\title{
Modelo matemático aplicado ao processo de extrusão de argilas
}

\section{(Mathematical model applied to the process of clay extrusion)}

\author{
F. A. Andrade, H. A. Al-Qureshi, D. Hotza \\ Núcleo de Pesquisa em Materiais Cerâmicos - CERMAT, Departamentos de Engenharia Mecânica - EMC e \\ Engenharia Química - ENQ, Universidade Federal de Santa Catarina - UFSC, Florianópolis, SC 88040-900 \\ hotza@enq.ufsc.br
}

Resumo

\begin{abstract}
As argilas, quando misturadas com água, adquirem a propriedade chamada plasticidade que tem papel fundamental durante o seu processamento. As técnicas empregadas na caracterização da plasticidade muitas vezes não revelam resultados quantitativos que permitam sua aplicação em modelos matemáticos do processo de conformação das argilas. Este trabalho teve por objetivo desenvolver um modelo matemático para cálculo da pressão média de extrusão, avaliando a plasticidade através de ensaios de compressão. Em seguida, os resultados teóricos da pressão de extrusão foram comparados aos resultados experimentais, sendo encontrado um bom acordo entre eles. Foi possível observar uma correlação entre tensão efetiva de compressão e pressão de extrusão. Para a equação que modela o processo de extrusão, a tensão efetiva obtida pelo ensaio de compressão foi um parâmetro significativo que revela o comportamento da argila durante o seu processamento.
\end{abstract}

Palavras-chave: argila, extrusão, plasticidade, modelamento.

Abstract

The clays when mixed with water acquire the property known as plasticity, which plays a key role during processing. The techniques used to characterize the plasticity often do not show quantitative results that allow its application in mathematical models of the forming process of clays. This study aimed to develop a mathematical model for calculating the average pressure of extrusion, evaluating the plasticity through compression tests. Then, the theoretical results of the extrusion pressure were compared to experimental results and a good agreement was found between them. It was possible to observe a correlation between effective stress of compression and extrusion pressure. For the extrusion process modeling equation, the effective stress obtained by compression test was a significant parameter that reveals the behavior of the clay during processing.

Keywords: clay, extrusion, plasticity, modeling.

\section{INTRODUÇÃO}

Existem poucas áreas na tecnologia cujo desenvolvimento tem encontrado tantos campos de aplicação como a extrusão. Inicialmente, era empregada apenas para materiais cerâmicos simples, mas hoje em dia a extrusãoé aplicada nas mais diversas áreas, como na indústria de polímeros, metalúrgica, química ou mesmo alimentícia [1]. Atualmente, muitos estudos nesta área vêm se concentrando na obtenção de cerâmicas avançadas a partir da extrusão [2]. Apesar disso, ensaios que predigam o comportamento da argila durante a extrusão, bem como o modelamento matemático de algumas de suas características, em especial, a plasticidade, tornam-se dificultados pelo fato de muitas variáveis estarem envolvidas e não haver consenso quanto à escolha do método a ser utilizado [3, 4]. A economia energética, a regularidade do fluxo e a pressão adequada de extrusão são fatores que dependem do tipo de fieira e da plasticidade da massa [5]. Até recentemente, a extrusão de pastas cerâmicas tem sido estudada e compreendida de modo limitado [6]. A razão disso é o fato de que, historicamente, a extrusão de massas cerâmicas estava restrita à produção de produtos de baixo custo da cerâmica vermelha que não requeriam rigoroso controle dimensional ou de qualidade [7].

Com base no método aplicado à teoria da plasticidade dos metais [8,9], pretende-se neste trabalho apresentar a aplicação de um modelo matemático para o cálculo da pressão de extrusão para argilas.

\section{REFERENCIAL TEÓRICO}

A extrusão consiste em forçar um material através de uma matriz com a geometria da seção transversal final pretendida. Nessa análise, assume-se que o estado estacionário seja alcançado, e assim, a pressão média de compressão por unidade de compressão é dada por:

$$
\left.\sigma_{\text {ave }}\right|_{\mu_{\mathrm{w}}=0}=1,5 \int_{0}^{\ln \frac{\mathrm{A}_{0}}{\mathrm{~A}_{\mathrm{f}}}} \bar{\sigma} \mathrm{d} \bar{\varepsilon}
$$


onde $\mu_{w}$ é o coeficiente de atrito entre a massa e a parede, $\bar{\sigma}$ é a tensão efetiva, $\bar{\varepsilon}$ é a deformação efetiva e 1,5 é o fator de cisalhamento, introduzido para corrigir as perdas de energia, principalmente por atrito e trabalho redundante que não estão inclusas no modelo.

Para prever teoricamente a pressão média de extrusão, a tensão de compressão da massa argilosa precisa ser conhecida através de ensaios de compressão. Isso foi tratado matematicamente para argilas [10] e pode ser expresso da seguinte forma:

$$
\sigma_{\mathrm{z}}=-\bar{\sigma} \exp \left(\frac{2 \mu\left(\mathrm{r}_{\mathrm{f}}-\mathrm{r}\right)}{\mathrm{h}}\right)
$$

onde $\bar{\sigma}_{\text {comp }}$ é a tensão efetiva de compressão, $h$ é a altura do corpo-de-prova, $R_{f}$ é o raio final e $r$ é o raio instantâneo.

A carga axial compressiva pode ser avaliada através de

$\mathrm{F}=\int_{0}^{\mathrm{y}_{\mathrm{f}}} 2 \pi \sigma_{\mathrm{z}} \mathrm{dr}$ e usando a equação $\mathrm{B}$ tem-se

$\mathrm{F}=-2 \pi \bar{\sigma}_{\text {comp }}\left[-\frac{\mathrm{h}}{2 \mu}\left(\mathrm{R}_{\mathrm{f}}+\frac{\mathrm{h}}{2 \mu}\right)+\frac{\mathrm{h}^{2}}{4 \mu^{2}} \exp \left(\frac{2 \mu}{\mathrm{h}} \mathrm{R}_{\mathrm{f}}\right)\right]$

A equação $C$ será utilizada para a determinação da carga em função da variação no diâmetro para cada massa argilosa no processo de compressão. Desta forma, uma melhor abordagem, em contraste com os outros métodos existentes, tais como o índice de plasticidade de Atterberg e de Pfefferkorn, é esperada para a obtenção de corpos cerâmicos com plasticidade otimizada para uma determinada aplicação [10-12].

O processo de extrusão de uma massa argilosa e o equilíbrio de uma parte infinitesimal do corpo é mostrado na Fig. 1. A pressão média na zona A é tirada da teoria e é dada por [13]:

$$
\mathrm{P}_{\text {ave }}=\bar{\sigma}_{\text {comp }} \frac{(1+\mathrm{B})}{\mathrm{B}}\left[\left(\frac{\mathrm{R}_{0}}{\mathrm{R}_{\mathrm{f}}}\right)^{2 \mathrm{~B}}-1\right]
$$

onde $B=\mu_{w} \cot \alpha$, $\alpha$ é o ângulo do semicone da matriz, $\mu_{w}$

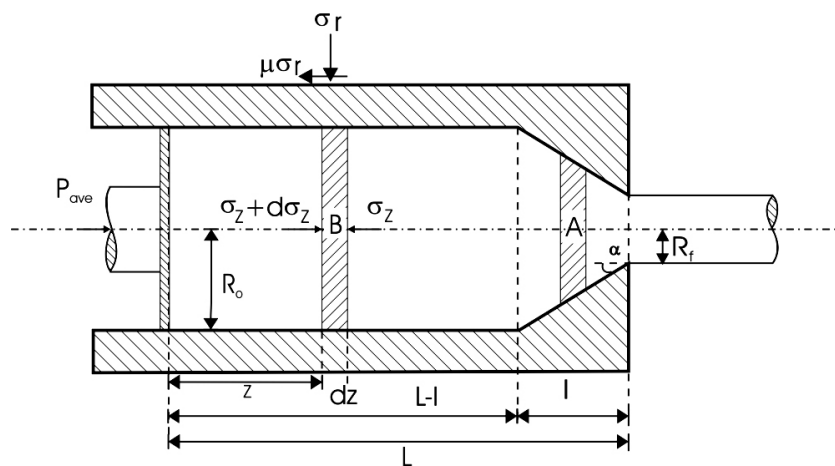

Figura 1: Diagrama esquemático do processo de extrusão através de uma matriz cônica.

[Figure 1: Scheme of the extrusion process through a conical die.] é o coeficiente de atrito entre massa argilosa e parede da extrusora e $\bar{\sigma}_{\text {comp }}$ é a tensão efetiva da massa argilosa no teste de compressão, equação B.

Por outro lado, o equilíbrio de forças na zona B pode ser facilmente criado:

$$
\pi R_{0}^{2}\left(\sigma_{z}+d \sigma_{z}\right)-\pi R_{0}^{2} \sigma_{z}-\mu_{w} \sigma_{r} 2 \pi R_{0} d z=0
$$

sendo

$$
\begin{aligned}
& \sigma_{y}=\bar{\sigma}+\sigma_{z} \\
& \ln \left(\bar{\sigma}+\sigma_{z}\right)=\frac{2 \mu_{w} Z}{R_{0}}+C
\end{aligned}
$$

Aplicando a condição de contorno para $\mathrm{z}=\mathrm{L}-\mathrm{l}$ :

$$
\sigma_{\mathrm{z}}=\bar{\sigma} \frac{(1+\mathrm{B})}{\mathrm{B}}\left[\left(\frac{\mathrm{R}_{0}}{\mathrm{R}_{\mathrm{f}}}\right)^{2 \mathrm{~B}}-1\right]
$$

A tensão média [11] pode ser dada por:

$$
\sigma_{\text {ave }}=\frac{\bar{\sigma}_{\text {comp }}}{\left(\mathrm{R}_{\mathrm{f}}-\mathrm{R}_{0}\right)} \frac{\mathrm{Z}}{2 \mu_{\text {comp }}}\left\{1-\exp \left[\frac{2 \mu_{\text {comp }}}{\mathrm{Z}}\left(\mathrm{R}_{\mathrm{f}}-\mathrm{R}_{0}\right)\right]\right\}
$$

Agora, com a expressão integrada colocando o valor z $=0$, e a pressão média de extrusão ideal dada na Equação I, a expressão final teórica para a pressão média de extrusão da massa argilosa através de uma matriz cônica pode ser determinada e é expressa como se segue:

$$
\begin{gathered}
\mathrm{P}_{\text {ave }}=\frac{\overline{\mathrm{O}}_{\text {comp }}}{\left(\mathrm{R}_{\mathrm{f}}-\mathrm{R}_{0}\right)} \frac{\mathrm{Z}}{2 \mu}\left\{1-\exp \left[\frac{2 \mu}{\mathrm{Z}}\left(\mathrm{R}_{\mathrm{f}}-\mathrm{R}_{0}\right)\right] *\right. \\
\left\{\left\{1+\left(\frac{(1+\mathrm{B})}{\mathrm{B}}\right)\left[\left(\frac{\mathrm{R}_{0}}{\mathrm{R}_{\mathrm{f}}}\right)^{2 \mathrm{~B}}-1\right]\right\} \exp \left\{2 \mathrm{~B}\left[-\frac{\mathrm{L}}{\mathrm{R}_{0}} \tan \alpha+\left(1-\frac{\mathrm{R}_{\mathrm{f}}}{\mathrm{R}_{0}}\right)\right]\right\}-1\right\}
\end{gathered}
$$

\section{MATERIAIS E MÉTODOS}

\section{Matérias-primas}

Foram selecionadas três argilas fornecidas pela empresa Paraná Mineração, denominadas A1, A2 e A3. Foram realizados ensaios para obter o limite de liquidez (LL) para cada argila, regido pela norma NBR 6459 (1984), bem como o limite de plasticidade (LP), segundo a norma NBR 7180 (1984). A preparação das amostras de pó para ambos os ensaios seguiu a norma NBR 6457 (1986).

\section{Comportamento da argila sob compressão}

Para cada composição de umidade do sistema águaargila o seu comportamento em um ensaio de compressão 
uniaxial foi avaliado com o objetivo de levantar os dados da tensão de compressão efetiva e também o valor do coeficiente de atrito, para aplicação no modelo teórico desenvolvido. Para esta etapa, as argilas foram desagregadas a seco em moinhos de bolas por 25 min e peneiradas em malha de $420 \mu \mathrm{m}$. Em seguida, para a determinação da umidade contida nas argilas, amostras contendo $10 \mathrm{~g}$ de material foram introduzidas em vidros relógio e levadas ao forno a $110 \pm 5{ }^{\circ} \mathrm{C}$ por $24 \mathrm{~h}$. Foram selecionados três valores de umidade, no intervalo compreendido entre LL e (LP+LL)/2, obtidos a partir do ensaio de Atterberg. Essas massas então foram preparadas e homogeneizadas misturando-se manualmente e posteriormente deixadas em repouso por $24 \mathrm{~h}$ em recipiente hermeticamente fechado, para homogeneização da umidade. Em etapa posterior, foram preparados os corpos-de-prova cilíndricos, mediante moldagem manual, em moldes de PVC com diâmetro de 17,0 mm e altura de 23,0 mm. Os corpos-deprova foram submetidos ao ensaio de compressão uniaxial em um texturômetro TA-XT2i (Stable Micro Systems), com velocidade de ensaio $0,1 \mathrm{~mm} / \mathrm{min}$ e célula de carga 25 kgf. O ensaio ocorreu até $50 \%$ de deformação. Para evitar distorções nas medidas do diâmetro e da altura, foi utilizada uma escala milimétrica como referência, foi posicionada ao lado do corpo-de-prova durante os ensaios. Com uma câmera digital (Canon SX110) foi efetuada a filmagem do ensaio, que foi sincronizada com os dados obtidos pelo texturômetro. Na sequiência, foram obtidas imagens a cada $15 \mathrm{~s}$ a partir da filmagem com um software (Windows Movie Maker). A mensuração dos diâmetros e altura dos corpos-de-prova foi realizada por meio de um software de análise de imagem (ImageTool). Com isso, foi possível obter os valores de raio e altura instantâneos e relacionar com a força aplicada, determinando-se assim os valores da tensão de compressão efetiva e o coeficiente de atrito, conforme equação $\mathrm{C}$.

\section{Comportamento da argila sob extrusão}

Para a extrusão, as argilas foram obtidas conforme descrito anteriormente, ou seja, foram secas, desagregadas, peneiradas e posteriormente foi adicionada água destilada para que as umidades finais fossem iguais às utilizadas no ensaio de compressão. Posteriormente, a massa foi passada em um laminador (Gelenski LIG-05) e então conformada em uma extrusora (Gelenski MVIG-05) para que pudessem ser obtidos os valores da pressão de extrusão para cada sistema água-argila selecionado. Para medição da pressão de extrusão foi instalado um manômetro entre o canhão da extrusora e o bocal intercambiável. O manômetro possui capacidade para medir até $20 \mathrm{kgf} / \mathrm{cm}^{2}$ e sua escala é graduada em $0,1 \mathrm{kgf} / \mathrm{cm}^{2}$.

\section{RESULTADOS E DISCUSSÃO}

\section{Composição química e mineralógica}

Os resultados da análise química por fluorescência de raios X são apresentados na Tabela I. A quantificação das fases foi realizada por análise racional [14] (Tabela II). O principal argilomineral nas três argilas é a caulinita. Para a argila $\mathrm{A} 3$, este corresponde a $\sim 85 \%$, enquanto o teor de quartzo, que atua como material não-plástico, corresponde a $\sim 13 \%$. Devido a esses valores, espera-se que esta argila desenvolva a maior plasticidade entre as três.

\section{Plasticidade pelo indice de Atterberg}

Os resultados dos ensaios para determinação do índice de plasticidade de Atterberg (IPA) para as argilas são apresentados na Tabela III. Observa-se a grande variação do IPA entre as argilas, que será útil para investigar a adequação do modelo matemático em condições variadas de plasticidade.

Tabela I - Composição química das argilas.

[Table I - Chemical composition of the clays.]

\begin{tabular}{cccccccccccc}
\hline Óxidos (\%) & $\mathrm{SiO}_{2}$ & $\mathrm{Al}_{2} \mathrm{O}_{3}$ & $\mathrm{Na}_{2} \mathrm{O}$ & $\mathrm{K}_{2} \mathrm{O}$ & $\mathrm{MgO}$ & $\mathrm{CaO}$ & $\mathrm{Fe}_{2} \mathrm{O}_{3}$ & $\mathrm{MnO}$ & $\mathrm{TiO}_{2}$ & $\mathrm{P}_{2} \mathrm{O}_{5}$ & $\mathrm{PF}$ \\
\hline $\mathrm{A} 1$ & 62,83 & 22,86 & 0,07 & 1,10 & 0,57 & 0,02 & 2,39 & 0,06 & 1,36 & 0,06 & 9,26 \\
$\mathrm{~A} 2$ & 61,57 & 25,08 & 0,01 & 0,48 & 0,13 & 0,02 & 0,38 & 0,01 & 0,42 & 0,05 & 8,37 \\
$\mathrm{~A} 3$ & 52,45 & 33,43 & 0,01 & 0,27 & 0,10 & 0,00 & 0,73 & 0,01 & 2,11 & 0,06 & 13,33 \\
\hline
\end{tabular}

PF: perda ao fogo

Tabela II - Composição de fases das argilas.

[Table II - Phase composition of the clays.]

\begin{tabular}{cccc}
\hline Fase (\%) & A1 & A2 & A3 \\
\hline Caulinita & 57,88 & 63,50 & 84,64 \\
Quartzo & 35,88 & 32,00 & 13,04 \\
Acessórios & 6,24 & 4,50 & 2,32 \\
\hline
\end{tabular}

Tabela III - Limites e índice de plasticidade de Atterberg. [Table III - Atterberg's plasticity limits and index.]

\begin{tabular}{cccc}
\hline Argila & LL $(\%)$ & LP $(\%)$ & IPA $(\%)$ \\
\hline A1 & 64,4 & 44,0 & 20,4 \\
A2 & 44,6 & 30,9 & 13,7 \\
A3 & 72,6 & 42,8 & 29,8 \\
\hline
\end{tabular}


Tabela IV - Resultados dos ensaios de compressão.

[Table IV - Compression test results.]

\begin{tabular}{ccccc}
\hline Argila & $\begin{array}{c}\text { Umidade } \\
(\%)\end{array}$ & Atterberg & $\begin{array}{c}\text { Tensão } \\
\text { efetiva de } \\
\text { compressão } \\
(\mathrm{kPa})\end{array}$ & $\begin{array}{c}\text { Coeficiente } \\
\text { de atrito } \\
\left(\mu_{\text {comp }}\right)\end{array}$ \\
\hline \multirow{4}{*}{ A1 } & 43,3 & LP & 71,0 & 0,15 \\
& 48,4 & média & 42,0 & 0,15 \\
& 54,1 & $($ LL+LP)/2 & 14,3 & 0,15 \\
\hline \multirow{3}{*}{ A2 } & 32,4 & LP & 80,0 & 0,15 \\
& 35,3 & média & 44,0 & 0,15 \\
& 38,6 & $($ LL+LP)/2 & 27,0 & 0,15 \\
\hline \multirow{3}{*}{ A3 } & 42,9 & LP & 62,0 & 0,15 \\
& 48,9 & média & 32,0 & 0,15 \\
& 56,5 & $($ LL+LP)/2 & 15,5 & 0,15 \\
\hline
\end{tabular}

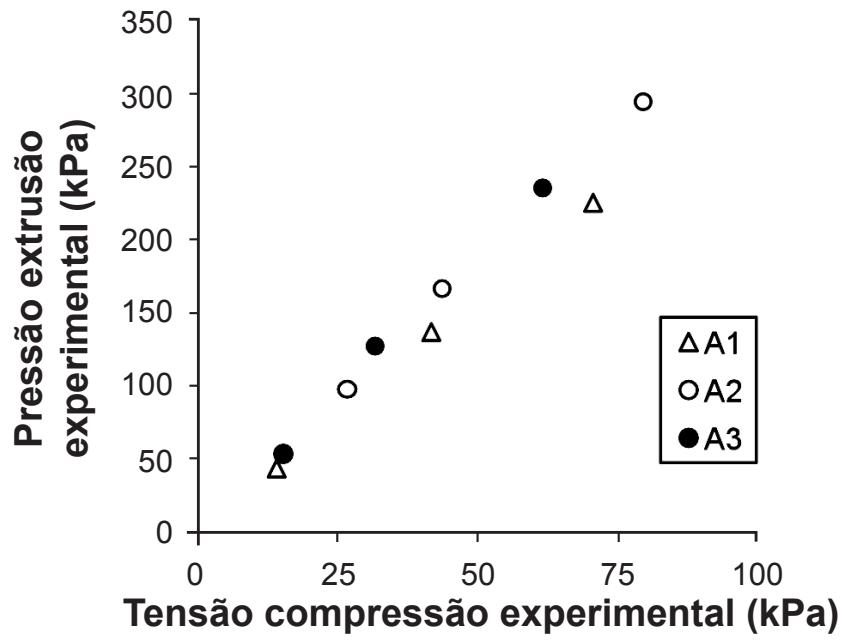

Figura 2: Correlação entre a pressão experimental de extrusão e a tensão efetiva de compressão.

[Figure 2: Correlation between the experimental extrusion pressure and the effective compression stress.]

Tabela V - Resultados da extrusão das argilas.

[Table V-Clay extrusion results.]

\begin{tabular}{cccccc}
\hline Argila & Umidade (\%) & $\begin{array}{c}\text { Pressão extrusão } \\
\text { Teórica }(\mathrm{kPa})\end{array}$ & $\begin{array}{c}\text { Pressão extrusão } \\
\text { Experimental }(\mathrm{kPa})\end{array}$ & $\begin{array}{c}\text { Diferença } \\
\text { Pressão Teórica e e } \\
\text { Experimental (\%) }\end{array}$ & $\begin{array}{c}\text { Coeficiente de } \\
\text { atrito parede }\left(\mu_{\mathrm{w}}\right)\end{array}$ \\
\hline \multirow{3}{*}{ A1 } & 43,30 & 224,8 & 225,4 & $-0,28$ & 0,56 \\
& 48,40 & 130,7 & 137,2 & $-4,77$ & 0,55 \\
& 54,10 & 43,7 & 44,1 & $-0,87$ & 0,54 \\
\hline \multirow{3}{*}{ A2 } & 32,35 & 297,4 & 294,0 & 1,15 & 0,65 \\
& 35,25 & 160,6 & 166,6 & $-3,59$ & 0,64 \\
& 38,63 & 96,8 & 98,0 & $-1,22$ & 0,63 \\
\hline \multirow{3}{*}{ A3 } & 42,90 & 239,0 & 235,2 & 1,62 & 0,67 \\
& 48,90 & 121,1 & 127,4 & $-4,92$ & 0,66 \\
& 56,50 & 57,6 & 53,9 & 6,90 & 0,65 \\
\hline
\end{tabular}

\section{Ensaio de compressão e extrusão}

A partir dos dados da carga em função do deslocamento gerados pelo texturômetro, puderam-se obter os dados dispostos na Tabela IV segundo a equação C.

Com os resultados de tensão efetiva e coeficiente de atrito obtidos nos ensaios de compressão, os mesmos foram inseridos na equação $J$ para o cálculo da pressão de extrusão teórica. Na Tabela V são apresentados os resultados experimentais da pressão de extrusão para os mesmos pontos de umidades dos ensaios de compressão. Nota-se um grande acordo entre os pontos experimentais e os previstos pela equação.

Dados referentes às condições de atrito entre uma massa cerâmica e a parede de equipamentos não são disponíveis facilmente na literatura. Apesar do coeficiente de atrito ser uma variável dinâmica, neste trabalho o mesmo foi considerado constante.
Na Fig. 2 pode ser observada a correlação entre a pressão experimental de extrusão e a tensão efetiva de compressão, obtida do ensaio de compressão. Há uma ótima correlação para todas as argilas, bem como quando os resultados são agrupados para todas as argilas, com um $\mathrm{R}^{2}$ de 0,97 . Concluise que o parâmetro tensão efetiva é um fator significativo na equação, e permite avaliar quantitativamente a pressão necessária durante a extrusão em função dos resultados obtidos nos ensaios de compressão.

Uma análise da Fig. 3 mostra que o raio final utilizado no bocal da extrusora influencia significativamente a pressão necessária à conformação das argilas. É possível determinar qual o diâmetro mínimo que poderia ser extrudado neste tipo de equipamento, levando-se em conta a pressão disponível no equipamento e a umidade da massa. Pode-se, portanto, com o ensaio de compressão, avaliar o possível comportamento da argila durante a extrusão, dependendo da sua umidade $\mathrm{e}$, se for o caso, indicar a melhor geometria do bocal para 


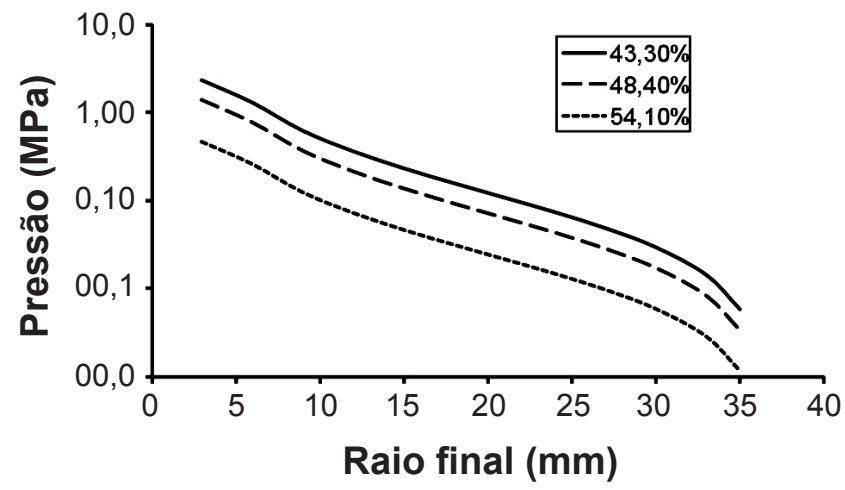

Figura 3: Pressão total em função do raio final para diferentes níveis de umidade. Extrusão da argila A1 em bocal cilíndrico. Raio inicial: $38 \mathrm{~mm}$.

[Figure 3: Total pressure as a function of the final radius for different moisture contents. Extrusion of Al clay. Initial radius: $38 \mathrm{~mm}$.]

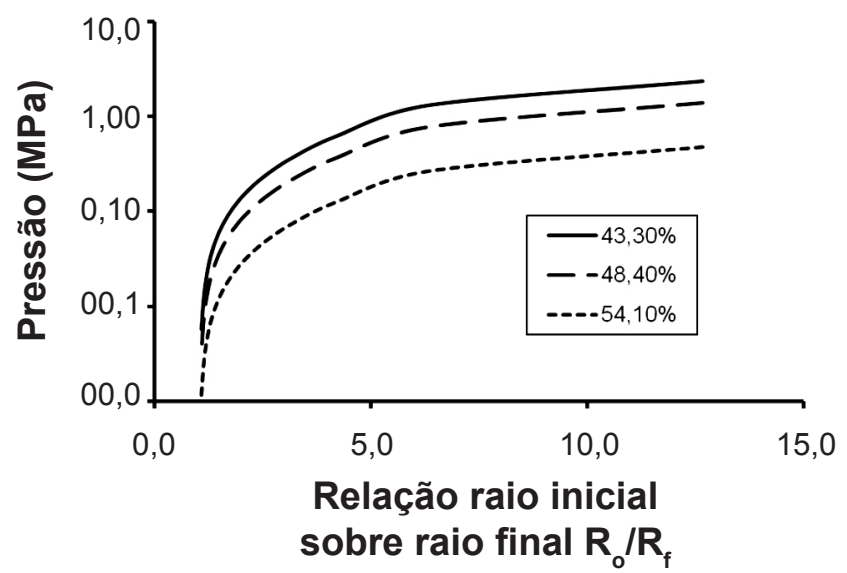

Figura 4: Pressão total em função da relação raio inicial/final para diferentes níveis de umidade. Argila A1.

[Figure 4: Total pressure as a function of the ratio final/initial radius for different moisture contents. A1 clay.]

sua conformação. Pode-se avaliar também o comportamento descrito anteriormente com auxílio das curvas mostradas na Fig. 4, correspondente ao comportamento da pressão de extrusão em função da relação raio inicial sobre raio final.

\section{CONCLUSÕES}

O sucesso para a produção de materiais tanto em escala laboratorial quanto na industrial requer um domínio sobre o tipo de processamento aplicado ao material, além do conhecimento acerca da matéria-prima e suas propriedades ou características. Com esse intuito, modelos teóricos que possam prever o comportamento do material antes do seu processamento são ferramentas úteis dentro do campo da Engenharia. Neste trabalho conseguiu-se aplicar um modelo matemático ao cálculo da pressão de extrusão de sistemas argilosos. Os resultados mostraram que este modelo pode ser empregado para argilas com variada gama de plasticidade. Foi possível averiguar que a tensão efetiva de compressão é um fator predominante na estimativa da pressão de extrusão, sendo encontrada uma ótima correlação entre esses parâmetros.

\section{AGRADECIMENTOS}

Ao $\mathrm{CNPq}$ pelo apoio financeiro e aos laboratórios da UFSC: PROFI/EQA, CERMAT/EMC e LMCC/ECV.

Abreviações e símbolos

$\mathrm{A}_{0}$ : área inicial; $\mathrm{A}_{\mathrm{f}}$ : área final; $\mathrm{F}$ : força externa aplicada, h: altura de corpo-de-prova; IPA: índice de plasticidade de Atterberg; L: comprimento total da extrusora; LL: limite de liquidez; LP: limite plástico; $\mathrm{P}_{\mathrm{m}}$ : pressão média de extrusão; $\mathrm{r}$ : raio instantâneo; $\mathrm{R}_{0}$ : raio inicial da extrusora; $\mathrm{R}_{\mathrm{f}}$ : raio final da extrusora; z: deslocamento do pistão de extrusão; $\alpha$ : semi-ângulo do bocal de extrusão; $\bar{\varepsilon}$ deformação efetiva; $\mu$ : coeficiente de atrito entre punção e massa; $\mu_{\mathrm{w}}$ : coeficiente de atrito entre parede da extrusora e massa; $\sigma_{r}$ : tensão radial; $\sigma_{z}$ : tensão axial; $\sigma_{\text {ave }}$ pressão média de extrusão; $\bar{\sigma}$ : tensão efetiva; $\bar{\sigma}_{\text {comp: }}$ tensão e fetiva de compressão.

\section{REFERÊNCIAS}

[1] J. Benbow, J. Bridgwater, Paste Flow and Extrusion, Oxford, Inglaterra (1993).

[2] F. Händle (Ed.), Extrusion in Ceramics, Springer, Berlin, Alemanha (2007).

[3] J. S. Reed, Principles of Ceramic Processing, $2^{\text {nd }}$ Ed., Wiley, New York, EUA (1995).

[4] M. Dondi, Ceram. Ind. 11, 3 (2006) 36.

[5] M. J. Ribeiro, A. A. L. Ferreira, J. A. Labrincha, Ceram. Ind. 8, 1 (2003) 37.

[6] A. S. Burbidge, J. Bridgwater, Chem. Eng. Sci. 50, 16 (1995) 2531.

[7] R. S. Macedo, R. R. Menezes, G. A. Neves, H. C. Ferreira, Cerâmica 54, 332 (2008) 411.

[8] B. Avitzur, Metal Forming: Process and Analysis, McGraw-Hill, New York, EUA (1968).

[9] N. R. Chitkara, A. Aleem, Int. J. Mechanical Sci. 43, 7 (2001) 1661.

[10] O. J. U. Flores, L. Mendes, A. L. G. Prette, M. C. Fredel, H. A. Al-Qureshi, D. Hotza, Anais $51^{\circ}$ Cong. Bras. Ceram., Salvador, BA (2007).

[11] H. A. Al-Qureshi, O. J. U. Flores, D. Hotza, Anais 52 Cong. Bras. Cerâm., Florianópolis, SC (2008).

[12] F. A. Andrade, H. A. Al-Qureshi, D. Hotza, Proc. $11^{\text {th }}$ Int. Conf. Adv. Mater., Rio de Janeiro, RJ (2009).

[13] G. Sachs, O. Hoffman, Introduction to the Theory of Plasticity for Engineers, McGraw-Hill, New York, EUA (1953)

[14] C. Coelho, N. Roqueiro, D. Hotza, Mater. Lett. 52, 6 (2002) 394.

(Rec.01/04/2010, Rev. 19/06/2010) 